\title{
DIVERSIDAD GENÉTICA DE LAS VARIEDADES DE ARROZ FLAR LIBERADAS ENTRE 2003-2014 ${ }^{1}$
}

\author{
Luis Eduardo Berrio-Orozco ${ }^{2}$, Édgar Alonso Torres-Toro ${ }^{3}$, Jairo Barona-Valencia ${ }^{3}$, Juan Bosco Cuásquer-Sedano ${ }^{3}$
}

\section{RESUMEN}

Diversidad genética de las variedades de arroz FLAR liberadas entre 2003-2014. El objetivo de este trabajo fue determinar la base genética, el coeficiente de parentesco y la diversidad genética de las variedades de arroz liberadas entre el 2003 - 2014 en trece países miembros del Fondo Latinoamericano para Arroz de Riego (FLAR). Para ello, se analizaron las genealogías de 51 variedades, en el Centro Internacional de Agricultura Tropical (CIAT), Palmira, Colombia, durante los años 2014 y 2015. La variabilidad genética de las variedades estuvo representada por 120 ancestrales (2,4 ancestral/variedad); 33 de estos contribuyeron con el $83,9 \%$ de sus genes, de los cuales tres (ancestrales que originaron IR8) aportaron el 35,6\% de sus genes. También se mostró que el coeficiente de parentesco $\left(\mathrm{r}_{\mathrm{xy}}\right)$ entre las diferentes variedades comerciales varió de 0,03 (muy poco relacionadas), hasta 0,99 (altamente relacionadas). El promedio de todas las 51 variedades fue de $\left(\mathrm{r}_{\mathrm{xy}}\right)$ 0,19. A nivel de variedades por país, se encontró que el promedio fue variable, el mínimo lo obtuvo Ecuador con 0,13, y el máximo fue de 0,31 para las variedades de Venezuela. El análisis de agrupamiento separó a los genotipos en catorce grupos distintos, donde existen materiales bastante relacionados y otros muy poco relacionados. Estos resultados muestran que se ha obtenido una ampliación de la base genética.

Palabras claves: genealogías, cultivares tradicionales, diversidad genética, progenitores, genes.

\begin{abstract}
Genetic diversity of the FLAR rice varieties released between 2003-2014. The objective of this study was to determine the genetic basis, the coefficient of kinship, and the genetic diversity of rice varieties released between 2003 and 2014, in thirteen member countries of the Latin America Fund for Irrigated Rice (FLAR). Pedigrees of o 51 rice varieties were analyzed at the International Center for Tropical Agriculture (CIAT), Palmira, Colombia between 2014-2015 The genetic variability of these varieties was represented by 120 ancestral or traditional cultivars $(2.4$ ancestral/variety); 33 of these contributed to the $83.9 \%$ of their genes, from which, three (ancestors with IR 8 origin), accounted for $35.6 \%$ of their genes. It was also shown that the coefficient of kinship $\left(\mathrm{r}_{\mathrm{xy}}\right)$ between different commercial varieties ranged from 0.03 (very unrelated), up to 0.99 (highly related). The average $r_{x y}$ of all 51 varieties was $=$ 0.19 . Regarding the varieties by country, the average $r_{x y}$ varied, the minimum was obtained by Ecuador 0.13 and the varieties from Venezuela obtained the maximum 0.31. The cluster analysis separated the genotypes in fourteen different groups based on highly related or very unrelated varieties. These results indicate that a broadening of the genetic base has been accomplished.
\end{abstract}

Keywords: pedigrees, traditional cultivars, genetic diversity, parents, genes.

\footnotetext{
Recibido: 30 de junio, 2015. Aceptado: 10 de noviembre, 2015. Este trabajo formó parte de la investigación que realiza el programa de mejoramiento del Fondo Latinoamericano de Arroz de Riego (FLAR).

2 Fondo Latinoamericano de Arroz de Riego (FLAR).Colombia. leberrio@gmail.com

3 Centro Internacional de Agricultura Tropical (CIAT),Programa de Arroz.Colombia.e.a.torres@cgiar.org, j.barona@cgiar.org, j.b.cuasquer@ cgiar.org
}

(C) 2016 Agronomía Mesoamericana es desarrollada en la Universidad de Costa Rica y se encuentra licenciada con Creative Commons Reconocimiento-NoComercial-SinObraDerivada 3.0 Costa Rica. Para más información escríbanos a pccmca@ucr.ac.cr 


\section{INTRODUCCIÓN}

La ampliación de la base genética o mejoramiento del germoplasma es fundamental, debido a que el mejoramiento intensivo moderno reduce la variabilidad genética, problema que se acrecienta más en especies que se auto-fecundan como en el caso del arroz (Wang et al., 1992). La estrecha variabilidad genética existente en las variedades de arroz modernas, las ha convertido en más vulnerables a estrés biótico y abiótico, lo cual explica la tasa más lenta de progreso genético alcanzada últimamente por los mejoradores (Tanksley y Nelson, 1996).

En América Latina, aún se menciona la estrecha base genética que se cree ha causado un supuesto techo de rendimiento en los materiales genéticos. En el programa de arroz del Centro Internacional de Agricultura Tropical (CIAT) se utilizó material genético japónica tropical de origen africano durante la década de los 80, principalmente entre 1984 y 1989. En un estudio realizado con la finalidad de determinar cambios en la variabilidad genética en el programa de arroz del CIAT entre los años 1998 y 1999 (Berrio-Orozco, 1999), se concluyó que a pesar de que se identificaron genotipos morfológica y molecularmente diferentes al tipo índica, la mayoría de las líneas desarrolladas entre cruces índica x japónica tuvieron un comportamiento similar al material tipo índica. Se considera que dichos resultados se explican por el hecho que los fitomejoradores se han concentrado en la selección de aquellos materiales con características similares a los tipos índica, propios de la región latinoamericana. Sin embargo, dicho autor encontró que se han incorporado algunas características propias de la subespecie japónica a los materiales latinoamericanos, como son el peso de mil granos, buena exerción de panícula, tolerancia a piricularia (Pyricularia grisea), al virus de la hoja blanca y estabilidad al centro blanco del grano.

La variabilidad genética en once variedades de arroz de riego liberadas en el año 1993 en siete países tropicales, evidenció que su base genética estaba dada por un total de 56 ancestrales (5,1 ancestral/ variedad) (Berrio-Orozco y Guimarães, 1995). De los 56 materiales, veintiséis contribuían con el 78,3\% de los genes, y la contribución de los tres ancestrales que originaron la variedad IR 8 suma el $24 \%$ de los genes, lo que indica una ganancia o ampliación de la base genética al compararla con los datos obtenidos por otros investigadores (Manosalva de Nivia et al., 1991; Cuevas-Pérez et al., 1992; Berrío-Orozco y Guimaraes, 1995).

Una forma de medir la diversidad que existe entre un grupo de materiales ha sido el coeficiente de parentesco $\left(r_{x y}\right)$, esta metodología discrimina entre pares de genotipos, midiendo la probabilidad de que dos genes tomados al azar, uno de cada individuo, lleven alelos idénticos por descendencia. Es la correlación entre los valores como progenitor o "breeding values", que representa la correlación que se esperaría si toda la varianza fenotípica fuera aditiva (Falconer y Mackay, 1996). El uso de $r_{x y}$ para estudios de diversidad ha sido difundido entre especies autógamas, tales como soya, trigo, cebada y recientemente en arroz. En soya se señaló que el $r_{x y}$ varía entre 0 y 1 , y que el cruzamiento entre progenitores con divergencia genética máxima $\left(r_{\mathrm{xy}}=\right.$ 0 ) es el que ofrece mayores oportunidades de obtener segregantes transgresivos (Vello et al., 1988). También se muestra que la correlación entre estimativas de la diversidad genética medida por $\mathrm{r}_{\mathrm{xy}}$ y por marcadores moleculares (RFLP) es baja en soya $(r=55 \%)$ y es alta en maíz ( $\mathrm{r}=93 \%$ ). Por lo que se concluyó que utilizar el $\mathrm{r}_{\mathrm{xy}}$ es más simple, rápido y económico que cualquiera de los otros métodos recomendados para estudiar la diversidad genética entre progenitores potenciales.

El conocimiento de la diversidad genética entre cultivares en una región es importante para planificar estrategias de mejoramiento y reducir la vulnerabilidad genética, debido a apariciones repentinas de plagas o enfermedades. Además, el conocimiento de los valores del coeficiente de parentesco $\left(r_{x y}\right)$ puede ser útil para la predicción de los valores como progenitor.

Con la restructuración del programa de arroz del CIAT en los 90s, el Fondo Latinoamericano para Arroz de Riego (FLAR) asumió la responsabilidad de la investigación aplicada del cultivo del arroz irrigado para la región en el año 1995. Hasta el año 2014, el trabajo colaborativo del FLAR y sus miembros, obtuvo como resultado 52 variedades liberadas en trece países miembros del FLAR en América Latina y el Caribe (LAC).

El objetivo de este trabajo fue determinar la base genética, el coeficiente de parentesco y la diversidad genética de las variedades de arroz liberadas entre el 2003 y 2014, en trece países miembros del Fondo Latinoamericano para Arroz de Riego (FLAR). 


\section{MATERIALES Y MÉTODOS}

Para la realización del presente estudio, se tomó toda la información suministrada por los técnicos de los países miembros del Fondo Latinoamericano para Arroz de Riego (FLAR), sobre las variedades liberadas para siembras comerciales, la cual fue registrada en la base de datos del FLAR en CIAT, Palmira, Colombia. El estudio se llevó a cabo durante los años 2014 y 2015.

El estudio comprendió el análisis de las genealogías de 52 variedades liberadas durante el periodo 2003-2014 en trece países de América Latina y el Caribe (LAC, Cuadro 1). Estas variedades provenían de un total de 37 cruces de tres líneas realizados por el FLAR a partir de 76 progenitores, de ellos 32 desarrollados por el FLAR (FL), veintiocho líneas del CIAT (CT y P), trece de programas regionales y tres líneas del IRRI (IR).

Del cruce FL05372 (dos en Costa Rica, tres en Venezuela, cuatro en Panamá y una en Ecuador) se originaron diez de estas variedades. Las variedadesliberadas de este cruce se caracterizan por expresar excelentes características morfológicas y

Cuadro 1. Variedades de arroz liberadas durante el periodo 2003-2014, en 13 países miembros del Fondo Latinoamericano para Arroz de Riego (FLAR). Colombia. 2014-2015.

Table 1. Rice varieties released between 2003-2014 in 13 member countries of the Latin American Fund for Irrigated Rice (FLAR). Colombia. 2014-2015.

\begin{tabular}{|c|c|c|c|c|c|}
\hline No. & Nombre & Pedigrí & Año & País & No. ancestros \\
\hline 1 & Tranquilo FL INTA & FL00222-18P-6-2P-MA-1A-13A & 2010 & Argentina & 57 \\
\hline 2 & Paititi & FL00144-1P-24-1P-M & 2004 & Bolivia & 65 \\
\hline 3 & Amboro & FL00468-5P-7-2P-M & 2004 & Bolivia & 66 \\
\hline 4 & CONARROZ FL 103 & FL04648-6P-9-1P-3P-M & 2008 & Bolivia & 54 \\
\hline 5 & MAC 18 & FL03188-7P-5-3P-3P-M & 2008 & Bolivia & 45 \\
\hline 6 & Saavedra 28 & FL03187-15P-3-3P-1P-M & 2009 & Bolivia & 70 \\
\hline 7 & Saavedra 44 & FL (DESCONOCIDO) & 2009 & Bolivia & - \\
\hline 8 & Corobicí & FL02066-4P-1-2P-M & 2006 & Costa Rica & 39 \\
\hline 9 & Palmar 18 & FL03160-6P-12-2P-1P-M & 2006 & Costa Rica & 50 \\
\hline 10 & Cabuyo & FL03784-13P-14-1P-1P-M & 2006 & Costa Rica & 69 \\
\hline 11 & Diria FL-15 & FL02063-7P-3-2P-3-M & 2009 & Costa Rica & 63 \\
\hline 12 & Cortez FL-48 & FL03187-12P-5-2P-3P-M & 2009 & Costa Rica & 70 \\
\hline 13 & Sierpe FL-250 & FL03323-5P-21-1P-1P-M & 2010 & Costa Rica & 67 \\
\hline 14 & Tenorio FL-238 & FL001028-8P-3-2P-1P-M-2X-3P-M & 2010 & Costa Rica & 43 \\
\hline 15 & Pasquiel FL-13 & FL05372-7P-4-3P-1P-M & 2010 & Costa Rica & 78 \\
\hline 16 & Curime FL-14 & FL05372-7P-4-4P-2P-M & 2010 & Costa Rica & 78 \\
\hline 17 & Garabito FL-163 & FL06747-4P-10-5P-3P-M & 2010 & Costa Rica & 74 \\
\hline 18 & Pasquiel 2 FL & FL06609-11P-12-1P-2P-M & 2014 & Costa Rica & 74 \\
\hline 19 & Jonhicuí FL & FL06609-20P-8-1P-1P-M & 2014 & Costa Rica & 74 \\
\hline 20 & Bu Cup FL & FL06733-16P-4-2P-2P-M & 2014 & Costa Rica & 88 \\
\hline 21 & FEDEARROZ 60 & FL03188-7P-5-3P-1P-M & 2007 & Colombia & 45 \\
\hline 22 & FEDEARROZ 174 & FL03174-8P-7-2P-2P-M & 2007 & Colombia & 56 \\
\hline 23 & FL FEDEARROZ 68 & FL08468 & 2014 & Colombia & 83 \\
\hline
\end{tabular}

Continúa $\ldots$ 
Continuación Cuadro 1...

\begin{tabular}{|c|c|c|c|c|c|}
\hline No. & Nombre & Pedigrí & Año & País & No. ancestros \\
\hline 24 & SENACA FL09 & FL03233-8P-6-2P-2P-M & 2009 & Ecuador & 45 \\
\hline 25 & SENACA FL 11 & FL05463-4P-13-1P-2P-M & 2011 & Ecuador & 75 \\
\hline 26 & SENACA FL 12 & FL05372-7P-7-2P-1P-M & 2012 & Ecuador & 78 \\
\hline 27 & INIAP FL 01 & FL07162-10P-5-3P-3P-M-EC & 2012 & Ecuador & 59 \\
\hline 28 & ICTA ARROZGUA 09 & FL03191-6P-4-1P-3P-M-1P-M & 2009 & Guatemala & 66 \\
\hline 29 & GRDB FL-10 & FL03199-2P-20-2P-1P-M & 2009 & Guyana & 64 \\
\hline 30 & GRDB FL-11 & Fl05482-8P-5-2P-1P-M & 2011 & Guyana & 52 \\
\hline 31 & FL0867 & FL00867-10P-15-3P-M & 2005 & Guy. Frac. & 67 \\
\hline 32 & DICTA Comayagua FL4-20 & FL0 4208-6P-5-2P-3P-M & 2011 & Honduras & 75 \\
\hline 33 & DICTA Playitas FL6-88 & FL0 6885-4P-3-2P-3P-M & 2011 & Honduras & 70 \\
\hline 34 & ANAR 2006 & FL01119-1P-5-2P-M & 2006 & Nicaragua & 53 \\
\hline 35 & ANAR 2012 FL & FL03160-6P-12-2P-1P-M & 2012 & Nicaragua & 50 \\
\hline 36 & IDIAP 145-05 & FL00144-1P-5-13P & 2005 & Panamá & 65 \\
\hline 37 & IDIAP 54-05 & FL00447-32P-3-1P-M & 2005 & Panamá & 67 \\
\hline 38 & IDIAP GAB 2 & FL03801-1P-1-1P-2P-M & 2010 & Panamá & 66 \\
\hline 39 & IDIAP FL 106-11 & FL07221-3P-3-3P-1P-M & 2011 & Panamá & 78 \\
\hline 40 & IDIAP FL137-11 & FL07321-3P-4-3P-1P-M & 2011 & Panamá & 68 \\
\hline 41 & Conagro 1 & FL05372-7P-1-3P-2P-M & 2011 & Panamá & 78 \\
\hline 42 & Conagro 2 & F105372-7P-4-4P-2P-M & 2011 & Panamá & 78 \\
\hline 43 & Conagro 3 & FL05372-7P-7-2P-1P-M & 2011 & Panamá & 78 \\
\hline 44 & Estrella FL 111 & FL06613-15P-10-1P-2P-M & 2012 & Panamá & 68 \\
\hline 45 & FCA 616 FL & FL05372-7P-5-3P-1P-M & 2013 & Panamá & 78 \\
\hline 46 & Jaragua FL & FL06786-1P-5-2P-2P-M & 2010 & Rep. Domin. & 77 \\
\hline 47 & Venezuela 21 & FL00147-8P-6-15P-M & 2003 & Venezuela & 69 \\
\hline 48 & Centauro & FL00984-8P-11-2P-2P-M-M & 2007 & Venezuela & 35 \\
\hline 49 & Pionero $2010 \mathrm{FL}$ & FL05372-7P-7-2P-M-1ASP & 2010 & Venezuela & 78 \\
\hline 50 & Payara 1 FL & FL05372-7P-4-5P-M-1PY & 2010 & Venezuela & 78 \\
\hline 51 & ASP 2012 FL & FL05372-7P-4-5P-M-2ASP & 2012 & Venezuela & 78 \\
\hline 52 & Soberana FL & FL03225-4P-5-1P-3P-M-1V & 2012 & Venezuela & 60 \\
\hline
\end{tabular}

buena tolerancia a enfermedades y buena calidad de grano. Asimismo, la variedad SAAVEDRA 28 de Bolivia, proviene del mismo cruce de la variedad Cortez FL 48 de Costa Rica. El cruce de FEDEARROZ 60 de Colombia es igual al de la variedad MAC 18 de Bolivia; la variedad Paititi de Bolivia proviene del mismo cruce de IDIAP 145-05 de Panamá; Palmar 18 de Costa Rica comparte el mismo pedigrí y cruce con
ANAR 2012 FL de Nicaragua, y dos de las últimas variedades liberadas en Costa Rica (Pasquiel 2 FL y Jonhiquí FL) son del mismo cruce.

La base genética de estas variedades y el coeficiente de parentesco se obtuvo al consultar la base de datos del Sistema Internacional de Información de Cultivos (ICIS acrónimo en inglés), en la implementación para arroz Sistema Internacional de Información de 
Arroz denominada "IRIS" (2011). Este sistema de datos está siendo desarrollado por varios centros de investigación del grupo consultivo para la investigación agrícola "CGIAR". Consiste en una base de datos computarizada de libre acceso a través de Internet, que permite un mejor manejo y utilización para el mejoramiento genético de la información relacionada con la genealogía, nomenclatura, evaluación y caracterización de germoplasma de arroz. Además, se utilizó la publicación sobre registros de cruzamientos realizados por el programa de arroz del Instituto Colombiano Agropecuario (ICA), del CIAT (1987), y la base de datos del FLAR. Para el análisis, el sistema fue alimentado con la genealogía y sus progenitores.

Base genética: para cada variedad fueron identificados los ancestrales presentes en su genealogía o pedigrí (Cuadro 1), el país de origen y la contribución genética, utilizando el comando .MGM en el módulo BROWSE del IRIS. La contribución genética (comando CONT)indica la probabilidad de que un alelo tomado al azar en un genotipo sea derivado de determinado ancestral. En cuanto que un ancestral corresponde a un cultivar terminal en el pedigrí, también conocido como "land race" con progenitores no conocidos y que no está emparentado con otros cultivares o ancestros; es decir, el coeficiente de endogamia es cero $(\mathrm{F}=0)$.

La contribución genética $(\mathrm{CG})$, corresponde a la proporción teórica de genes que vienen de un ancestral después del análisis de su genealogía o pedigrí ya que se asume que cuando un cruce es hecho, $50 \%$ de los genes vienen de cada uno de los progenitores.

La contribución genética total (CGT) de un ancestral fue determinada por la suma de su CG en todas las variedades estudiadas. Posteriormente, se calculó la contribución genética relativa de un ancestral (CGR\%) en relación con la suma de las CGT's de este, dividido por el número de veces que se repite en las 51 variedades estudiadas. Los ancestrales fueron ordenados de forma decreciente en cuanto a su CGR, la suma sucesiva de dichas contribuciones, generó la contribución genética acumulada (CGA). Igualmente, fue contado el número de veces que un determinado ancestral participó en las variedades estudiadas, este valor fue utilizado para calcular la frecuencia de participación relativa (FPR\%), que representa el porcentaje de las variedades en que un ancestral está presente.
Coeficiente de parentesco: para estudiar la diversidad genética entre las variedades comerciales de arroz liberadas en América Latina y el Caribe durante el periodo 2003-2014, se analizaron los pedigríes (Cuadro 1) y los respectivos progenitores de las 51 variedades (no se incluyó la variedad SAAVEDRA 44 por desconocerse su pedigrí). Estos materiales tienen y comienzan a tener áreas cultivadas importantes en los diferentes países, por ejemplo: las variedades de Panamá IDIAP 54-05 e IDIAP 145-05 con un 42\% del área, Palmar 18 de Costa Rica y Mac 18 de Bolivia ocupan un área del 60\%, respectivamente. La variedad Jaragua FL es actualmente la primera de República Dominicana y ocupa más del $40 \%$ del área cultivada.

La información de la genealogía o pedigrí fue utilizada para calcular el coeficiente de parentesco $\left(\mathrm{r}_{\mathrm{xy}}\right)$ para todos los pares de combinaciones de los 51 genotipos, utilizando el comando .cop del módulo BROWSE del programa IRIS.

Los supuestos que utiliza el programa son: a) genotipos homocigotos, b) genotipos sin ancestrales comunes no están relacionados y c) los progenitores contribuyen igualmente a la progenie.

En un sentido general, esta es una medida de la ascendencia común entre dos genotipos y de la diversidad genómica latente. Representa la similaridad genética y se calcula utilizando la ecuación (1), Falconer y Mackay (1996).

$$
r_{x y}=\frac{2 \theta_{x y}}{\sqrt{\left(1+F_{X}\right)\left(1+F_{Y}\right)}}
$$

Donde $\theta_{x y}$ es el coeficiente de coascendencia y $F_{x}$ y $F_{y}$, son los coeficientes de consanguinidad de X y Y, respectivamente. Por definición, un genotipo tiene un $\mathrm{r}_{\mathrm{xy}}$ de 1,00 consigo mismo, en progenies de hermanos completos $r_{x y}$ es igual a 0,50 , en progenies de medios hermanos $r_{x y}=0,25$ y en individuos que no comparten progenitores $r_{x y}=0,00$. Para construir el dendograma se utilizó como medida de similaridad el coeficiente de parentesco, como lo mencionaron Gopal y Oyama (2005). Los materiales fueron agrupados mediante el uso del método de agrupamiento de a pares, no corregido utilizando promedios aritméticos (UPMGA), el dendograma fue generado utilizando la función TREE PLOT del programa NTSYSpc (Rohlf, 1997). 


\section{RESULTADOS Y DISCUSIÓN}

Base genética: el análisis de la genealogía de las 51 variedades de arroz estudiadas (Cuadro 1), mostró que un total de 120 ancestrales contribuyen con la base genética del arroz de riego en la región. Esto da un promedio de 2,4 ancestral/variedad. En el Cuadro 2, se indican los nombres de dichos ancestrales, origen y su contribución. El 45,8\% de estos (55) fueron originados en Asia. Dichos ancestrales aportan un total de $74 \%$ de los genes; entre estos, el mayor número procede de la India con doce ancestrales, seguido de Filipinas con once, Taiwán con siete, Tailandia con seis y cuatro son de Japón. Entretanto, el 19,2\% (veintitrés ancestrales) son originarios del continente americano con un aporte total del 9,7\% de sus genes (Estados Unidos y Brasil con seis ancestrales, cada uno, Surinam con cinco, Guyana con dos, y Costa Rica, Chile, Honduras y México aportan solo uno). Otros 7,5\% (nueve ancestrales) son de origen africano con un aporte total del 7,4\% de sus genes (Zaire y Nigeria con dos cada uno, y con uno están: Senegal, Madagascar, Guinea, Nigeria, Liberia y Costa De Marfil). Dos ancestrales son de origen europeo (Italia). Un porcentaje grande, del $25,8 \%$ es ocupado por ancestrales de origen desconocido (31) y aportan un total del 7,7\% de sus genes. La predominancia de germoplasma asiático, principalmente originado en India y Filipinas, probablemente es el resultado de la selección para condiciones de arroz de riego y las preferencias de consumo que se orientan hacia arroces de grano largo con amilosa alta; además, en esas regiones se localizan los centros primarios y secundarios de diversidad del arroz.

A nivel de cada variedad (Cuadro 1) se mostró el número de ancestrales que hacen parte en el desarrollo de cada una de las variedades. Se observó que dicho número varió de 35 ancestrales para la variedad Centauro de Venezuela, a 88 para la variedad Bu Cup FL, liberada por SENUMISA, Costa Rica en 2014. También se encontró un total de 83 ancestrales para la variedad FL FEDEARROZ 68 liberada en el 2014 por FEDEARROZ, Colombia. La diferencia en el número de ancestrales se refleja en la participación de los tres que originaron la variedad IR 8; mientras la variedad Centauro tiene un $41 \%$ de participación de IR 8, en Bu Cup FL se disminuye a $37 \%$ y en la variedad FL FEDEARROZ 68, dicho aporte es del 29\%. Lo anterior destaca los esfuerzos de los mejoradores de arroz en involucrar un mayor número de progenitores en busca de una ampliación de la variabilidad genética en las nuevas variedades, para así ampliar la estrecha base genética reportada y citada anteriormente.

Entre los 120 ancestrales que aportan el 99,99\% de los genes en las variedades de riego en la región, diecisiete de ellos tienen una frecuencia de participación relativa (FPR) de 100\%; es decir, están presentes en todas las variedades estudiadas. Dee Geo Woo Gen, Latil Saill y Cina, son los que dieron origen a la variedad IR8. Estos resultados concuerdan con lo que se menciona acerca de que más de un tercio de las variedades liberadas en América Latina utilizan a IR8 como progenitor, o líneas descendientes de este para su desarrollo (Cuevas-Pérez et al., 1992). En las variedades de arroz de riego en Brasil están presentes estos tres ancestrales en una frecuencia promedio de participación relativa de $86 \%$, lo que demuestra la estrecha relación genética entre las variedades de arroz bajo riego (Rangel et al., 1996). El cultivar IR8 inició la era del tipo moderno de planta para arroz de riego, representada por baja altura de planta, alto macollamiento, tallos fuertes y hojas erectas (Cuevas-Pérez et al., 1992).

Los datos presentados en el Cuadro 3 se refieren a la contribución genética relativa (CGR) y acumulada (CGA) de los diferentes ancestrales de mayor contribución en las 51 variedades estudiadas. Se observa que la base genética del arroz en la región, está representada por 33 ancestrales que aportan una contribución genética acumulada (CGA) del 83,9\% de genes. Entretanto, los diecisiete ancestrales presentes en todas las 51 variedades contribuyen con el $60,6 \%$ del conjunto génico. De estos, los tres progenitores de IR8 aportan el 35,6\% de los genes. Cuando son considerados únicamente aquellos materiales que aportan más del $2 \%$ de los genes, este número se reduce a once ancestrales y la CGA es de 59\%.

Estos resultados permiten inferir que la base genética del arroz actual de las variedades liberadas por miembros del FLAR en LAC, es menos estrecha que la base genética del arroz en América Latina y más amplia a la del arroz de riego en Brasil. En América Latina, se estudió un total de 143 variedades, se encontró un total de 101 ancestrales, de los cuales veintidós de ellos contribuían con el $83 \%$ de los genes y catorce estaban presentes en todas las variedades liberadas, con una contribución del $70 \%$ de sus genes. 
Cuadro 2. Número de ancestrales de arroz, origen y su contribución a la base genética de las 51 variedades liberadas durante el periodo 2003-2014, en 13 en países miembros del Fondo Latinoamericano para Arroz de Riego (FLAR). Colombia. 2014-2015.

Table 2. Number and origin of ancestors of rice, and their contribution to the genetic basis of the 51 released varieties between 2003-2014 in 13 member countries of the Latin American Fund for Irrigated Rice (FLAR). Colombia. 2014-2015.

\begin{tabular}{|c|c|c|}
\hline Continente / País & Ancestral & $\begin{array}{c}\text { Total } \\
\text { ancestrales }\end{array}$ \\
\hline \multicolumn{2}{|c|}{ ASIA (74\% contribución) } & 55 \\
\hline Bangladesh & Badkalamkati 65 & 1 \\
\hline China & 29 Lu 1, Cina y Gpno 1769 & 3 \\
\hline Indonesia & Bayang y Benong & 2 \\
\hline India & $\begin{array}{l}\text { Anaikomban, Arc 6650, Evarapandi, Gowdalu, Kitchili Samba, Latisail, Mudgo, } \\
\text { Pankhari 203, Thekkan, Unknown (India), Vellaikar, y Co } 13 .\end{array}$ & 12 \\
\hline Japón & Bozu, Joshyu, Kameji y Kiryousshi & 4 \\
\hline Corea & Chow Sung & 1 \\
\hline LKA & Babawee y H 105 & 2 \\
\hline Malasia & TR & 1 \\
\hline Pakistán & Basmati $370, \mathrm{Rb} 2$ y Red Rice & 3 \\
\hline Filipinas-IRRI & $\begin{array}{l}\text { Tadukan, Marong Paroc, C74 ,560, Sinawpagh, Carreon, W1543, Seraup Besar15, } \\
\text { D 52-37,Nira y Fb } 24\end{array}$ & 11 \\
\hline Tailandia & Khao Dawk Mali,Nam Sagui,Gam Pai,Leuang Tawn, Gsiam 29, Nahng Mon & 6 \\
\hline Taiwán & $\begin{array}{l}\text { Dee Geo Woo Gen,Igt, Oluan Chu,Pa Chiam,Taichung 176,Takao Iku 18,Tsai Yuan } \\
\text { Chung }\end{array}$ & 7 \\
\hline Vietnam & Tetep y Mong Chim Vang A & 2 \\
\hline \multicolumn{2}{|c|}{ America $(9,7 \%$ contribución) } & 23 \\
\hline Brasil & Dourado Precoce, Perola, Pratao, Iguape Cateto, Pasarinho y Batataes & 6 \\
\hline Chile & Oro & 1 \\
\hline Costa Rica & Palmira 105 & 1 \\
\hline Guyana & D $85-42$ y Machique & 2 \\
\hline Honduras & Honduras & 1 \\
\hline México & Jojutla & 1 \\
\hline Surinam & Costa Rica, Sml 80-5, Sml 81A, Sml 997 y Sml 1010 & 5 \\
\hline USA & $\begin{array}{l}\text { Hill Sel, Bruinmissie, Carolina Gold, Chinese, Desconocido (Japonese) y Improved } \\
\text { Blue Rose }\end{array}$ & 6 \\
\hline \multicolumn{2}{|c|}{ África $(7,4 \%$ contribución) } & 9 \\
\hline Costa de Marfil & $63-105$ & 1 \\
\hline Madagascar & Makalioka & 1 \\
\hline Guinea & Moroberekan & 1 \\
\hline Senegal & Dissi Hatif & 1 \\
\hline Zaire & Os 6 y Variety From Zaire & 2 \\
\hline Nigeria & Tox 939-107-2-101-1-1B, Rock 1 & 2 \\
\hline Liberia & Lac 23 (Red) & 1 \\
\hline \multicolumn{2}{|c|}{ Europa $(0,2 \%$ contribución) } & 2 \\
\hline Italia & Agostano y Balilla & 2 \\
\hline \multicolumn{2}{|c|}{ Desconocido (7,7\% contribución) } & 31 \\
\hline Desconocido & $\begin{array}{l}\text { Bg 6812, <Unknown>, Linawang, Venezuela 503, Aikoko, Vni Ir 3223, Dourado } \\
\text { Precoce 689, Lita 506, Malunja, Coll 6850, Ngovie } 20 \text { Slrs, 12-30, Ai Jiao Gen, } \\
\text { 77-5-3-4, 1943-1-1P-1B, } 419 \text { C-57, B1 681, B122, Carolino, SmlWashabo, Se } 363 \\
\text { G, Tchen Tchou Ai, Siam 25, Guinosgar, Tos 2578-7-4-2-3-B2, Lg Ag, O. Nivara, } \\
\text { Jagaari, Yola, Pachchaperumal y Vellai Illankalayan. }\end{array}$ & \\
\hline Total & & 120 \\
\hline
\end{tabular}


Cuadro 3. Contribución genética relativa (CGR\%), contribución genética acumulada (CGA\%) y frecuencia de participación relativa (FPR\%) de los 33 ancestrales de mayor contribución en las 51 variedades de arroz liberadas durante el periodo 2003-2014, en 13 países miembros del Fondo Latinoamericano para Arroz de Riego (FLAR). Colombia. 2014-2015.

Table 3. Relative genetic contribution (CGR\%), accumulated genetic contribution (CGA\%) and relative participation frequency (FPR\%) of the 33 ancestors of greatest contribution in the 51 rice varieties released between 2003-2014 in 13 member countries of the Latin America Fund for Irrigated Rice (FLAR). Colombia. 2014-2015.

\begin{tabular}{llccc}
\hline No. & Ancestrales & CGR\% & CGA\% & FPR\% \\
\hline 1 & Dee Geo Woo Gen & 13,04 & 13,04 & 100,00 \\
2 & Latisail & 11,34 & 24,38 & 100,00 \\
3 & Cina & 11,17 & 35,55 & 100,00 \\
4 & Makalioka & 4,16 & 39,71 & 96,08 \\
5 & Tadukan & 3,50 & 43,20 & 100,00 \\
6 & Takao Iku 18 & 3,46 & 46,66 & 100,00 \\
7 & Khao Dawk Mali & 2,72 & 49,39 & 100,00 \\
8 & Marong Paroc & 2,64 & 52,03 & 100,00 \\
9 & Batataes & 2,57 & 54,59 & 78,43 \\
10 & Tetep & 2,13 & 56,72 & 100,00 \\
11 & Pa Chiam & 2,04 & 58,77 & 100,00 \\
12 & Igt & 1,82 & 60,59 & 100,00 \\
13 & Mong Chim Vang A & 1,82 & 62,41 & 100,00 \\
14 & Palmira 105 & 1,73 & 64,14 & 100,00 \\
15 & <Unknown> & 1,73 & 65,87 & 86,27 \\
16 & Kiryouyoshi & 1,62 & 67,49 & 96,08 \\
17 & Dissi Hatif & 1,53 & 69,02 & 94,1 \\
18 & Sml 1010 & 1,51 & 70,53 & 80,39 \\
19 & Basmati 370 & 1,45 & 71,98 & 33,33 \\
20 & C 74 & 1,38 & 73,37 & 100,00 \\
21 & Nam Sagui & 1,33 & 74,70 & 80,39 \\
22 & Kitchili Samba & 1,07 & 75,77 & 98,04 \\
23 & Kameji & 1,04 & 76,81 & 96,08 \\
24 & Oluan Chu & 1,04 & 77,85 & 96,08 \\
25 & Bg 6812 & 1,00 & 78,84 & 52,94 \\
26 & Benong & 0,85 & 79,70 & 96,08 \\
27 & Gam Pai & 0,85 & 80,54 & 86,27 \\
28 & Tsai Yuan Chung & 0,81 & 81,36 & 100,00 \\
29 & Sml 997 & 0,78 & 82,14 & 84,31 \\
30 & Eravapandi & 0,74 & 82,88 & 96,08 \\
31 & Unknown (Japanese) & 0,63 & 83,51 & 100,00 \\
32 & Sinawpagh & 0,23 & 83,74 & 100,00 \\
33 & Hill Sel & 83,87 & 100,00 \\
\hline & & &
\end{tabular}

Los ancestrales que dieron origen a IR8 contribuyeron con el 39\% de los genes (Cuevas-Pérez et al., 1992). $\mathrm{Al}$ agrupar solamente los ancestrales que contribuyeron con más del $2 \%$ de los genes, se encontró que solo diez ancestrales contribuyeron con $68 \%$ del conjunto génico (Rangel y et al., 1996).

El aporte de los ancestros que originaron el IR8 en promedio (35\%), fue del $10 \%$, menor valor que cuando se comparó con los reportados para las variedades de América Latina y Brasil que fueron del $39 \%$ y $38 \%$, respectivamente. También cuando se comparó los veintidós ancestrales (base genética del 83\%) reportados por Cuevas-Pérez et al. (1992), se observó que solo catorce de ellos son comunes a la base genética de los 33 ancestrales encontrados en este estudio $(83,9 \%)$. Los diecinueve ancestrales nuevos aportaron un total de $26 \%$ de sus genes. La disminución de este aporte de IR8, y el cambio en la participación de algunos ancestrales donde algunos la incrementan y otros la disminuyen, es debido al proceso de selección de genotipos para la adaptación a determinados ambientes en la región.

La variabilidad genética de las 51 variedades liberadas durante el periodo 2003-2014 en trece países miembros del FLAR, está representada por 120 ancestrales (2,4 ancestral/variedad), de los cuales solo 33 ancestrales contribuyeron con el 83,9\% de sus genes, donde tres de ellos, que originaron la variedad IR8 aportan el 35,6\% de sus genes. De acuerdo a los anteriores resultados se considera que con la liberación de estas nuevas variedades de arroz, muchas de las cuales están siendo cultivadas en los campos de los agricultores, se ha obtenido una ganancia o una ampliación de la base genética en la región, cuando se compara con otros resultados, por ejemplo: 2,7 ancestral/variedad, con un 50\% de IR8, 1,6 ancestral/ variedad, con $49 \%$ de IR8, 0,7 ancestral/variedad y veintidós ancestrales aportando el $83 \%$ de sus genes, donde IR8 aporta el 39\%, y 3,1 ancestral/variedad con un 47,7\% de sus genes de IR8 (González-Tous et al., 1991; Manosalva de Nivia et al., 1991; Cuevas-Pérez et al., 1992; Acevedo et al., 2007). Se observa una tendencia general de involucrar un mayor número de ancestrales en las nuevas variedades (Cuadro 1). Esto es importante para el mejoramiento genético, ya que permitirá lograr mayor ganancia genética y posiblemente reducirá la vulnerabilidad a factores adversos. La diferencia en el número de cultivares 
se refleja en la participación de los tres ancestrales que originaron la variedad IR8, ya que la variedad Centauro (35 ancestrales) tiene un $41 \%$ de IR8, la nueva variedad FL FEDEARROZ 68 (83 ancestrales), tiene un $29 \%$ y las diez variedades del cruce FL05372 (78 ancestrales), tienen un 35\% de IR8.

El coeficiente de parentesco: este estudio mostró que el coeficiente de parentesco $\left(\mathrm{r}_{\mathrm{xy}}\right)$ entre las diferentes variedades comerciales varió de 0,03 (muy poco relacionadas) entre la variedad SENACA FL 9 de Ecuador y FEDEARROZ 174 de Colombia, hasta 0,99 (altamente relacionas) entre las variedades Curime FL 14 de Costa Rica y CONAGRO 2 FL de Panamá, entre SENACA FL 12 de Ecuador y CONAGRO 3 FL de Panamá, y entre las variedades ANAR 2012 FL de Nicaragua y Palmar 18 de Costa Rica (Cuadro 4). El promedio del coeficiente de parentesco $\left(r_{x y}\right)$ de las 51 variedades en estudio fue de 0,19 . En total se econtraron 1275 pares de combinaciones entre todas las 51 variedades estudiadas.

Entre ellas, 54 pares de combinaciones $(4,2 \%)$ entre variedades altamente relacionadas (más del $50 \%$ de sus genes). En este conjunto se encuentran veinticuatro variedades: diez de ellas provenientes del mismo cruce (FL05372), donde las cuatro variedades altamente relacionadas (arriba mencionadas) provienen de la misma genealogía, al igual que las variedades ANAR 2012 FL y Palmar $18\left(\mathrm{r}_{\mathrm{xy}}=0,99\right)$. Dos variedades Fedearroz 60 y MAC18 son hermanas $(0,89 \%)$, Saavedra 28 y Cortez FL $48\left(r_{x y}=0,56\right)$ son del mismo cruce, pero provienen de selecciones diferentes a partir de la generación F2, IDIAP FL 10611 y FEDEARROZ $174\left(\mathrm{r}_{\mathrm{xy}}=0,55\right)$, donde la IDIAP FL 106, tiene como tercer progenitor a la variedad FEDEARROZ 174, y las dos variedades de Costa Rica, Jonhicui FL y Pasquiel 2 FL $\left(r_{x y}=0,50\right)$, son del mismo cruce, pero provienen de selecciones diferentes en la generación F2 (Cuadro 1).

Existen otras relaciones intermedias del coeficiente de parentesco que varían entre 0,25 y 0,50 . Dentro de este grupo, 80 pares de combinaciones $(6,3 \%)$ comparten al menos uno de sus progenitores en su pedigrí o genealogía. La mayoría de pares de combinaciones (1141), que equivale al 89,5\%, entre las variedades estudiadas, estuvieron por debajo de un $r_{x y}$ del 0,25 y dentro de este conjunto 111 pares de ellas mostraron estar poco relacionadas entre sí, compartiendo menos del 10\% de sus genes.
A nivel de país, se analizaron los coeficientes de parentesco promedio de las variedades de Bolivia, Costa Rica, Colombia, Ecuador, Panamá y Venezuela. Los datos encontrados en Costa Rica del $r_{x y}=0,20$ son menos de la mitad al publicado por CuevasPérez et al. (1992), de 0,41, lo cual indica que el nivel de consanguinidad ha disminuido; es decir, se ha incrementado la variabilidad entre las nuevas variedades liberadas en Costa Rica. Estos resultados fueron también menores para las variedades de Ecuador $\left(r_{x y}=0,13\right.$ vs 0,28, según Cuevas-Pérez et al., 1992). En Bolivia los resultados fueron similares $(0,17$ vs $0,13)$, manteniéndose la misma consanguinidad entre las variedades. Igualmente para Panamá $(0,26$ vs 0,24$)$. Contrario a lo anterior, en Venezuela se encontró un $r_{x y}$ de 0,31, más alto al reportado por Cuevas et al. (1992), que fue de 0,13. Este último resultado posiblemente se debe a que del total de variedades estudiadadas en este periodo, el $50 \%$ provienen de un mismo cruce. Acevedo et al. (2007) estudiando diecinueve variedades de Venezuela, encontraron un $\mathrm{r}_{\mathrm{xy}}=0,20$.

La diversidad genética teórica y las interrelaciones entre las variedades de arroz liberadas en LAC, son mostradas más claramente a través del dendograma obtenido del análisis de agrupamiento (Figura 1). Esta figura está basada en similaridad medida por el coeficiente de parentesco, por tanto, cuanto mayor sea este valor, más similar genéticamente es un par o grupo de genotipos. Tomando como referencia un valor de similaridad de 0,20, los genotipos estudiados fueron separados en catorce grupos distintos (Cuadro 5).

Las variedades de arroz estudiadas constituyen un grupo heterogéneo de materiales de acuerdo con su parentesco, existen materiales bastante relacionados y otros muy poco relacionados. De esta forma se puede observar que el grupo I contiene variedades bastante relacionadas, resultados obvios, puesto que son del mismo cruce (FL05372) y algunas con la misma genealogía. Asimismo, se encontraron ocho variedades (grupos VII al XIV) muy poco relacionadas con las demás variedades.

De acuerdo con los anteriores resultados, se considera que con la liberación de estas 51 nuevas variedades de arroz en trece países miembros de FLAR, se ha obtenido una ganancia o una ampliación de la base genética del arroz en la región. En Costa Rica se encontró que el nivel de consanguinidad de sus variedades ha disminuido notablemente, es 


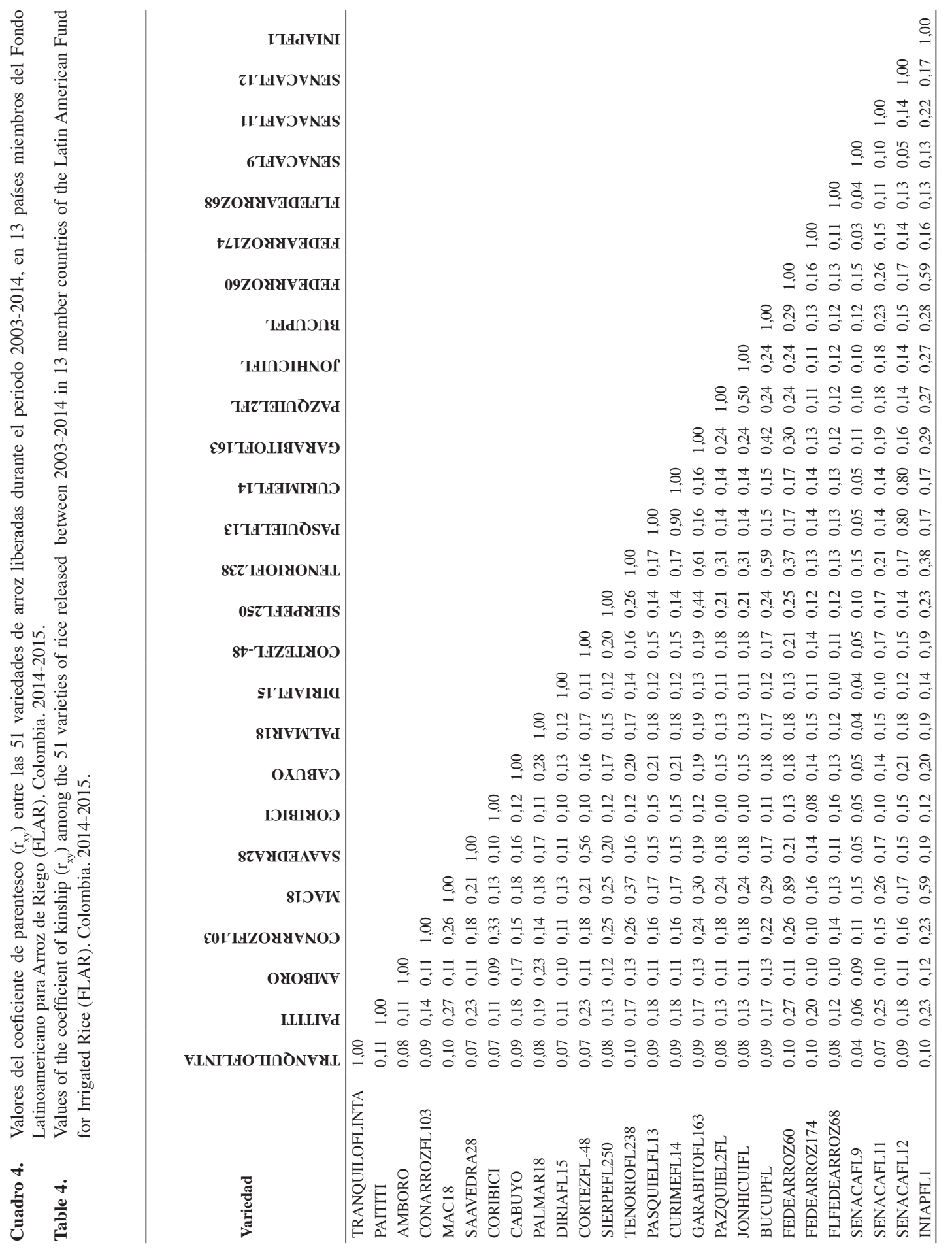




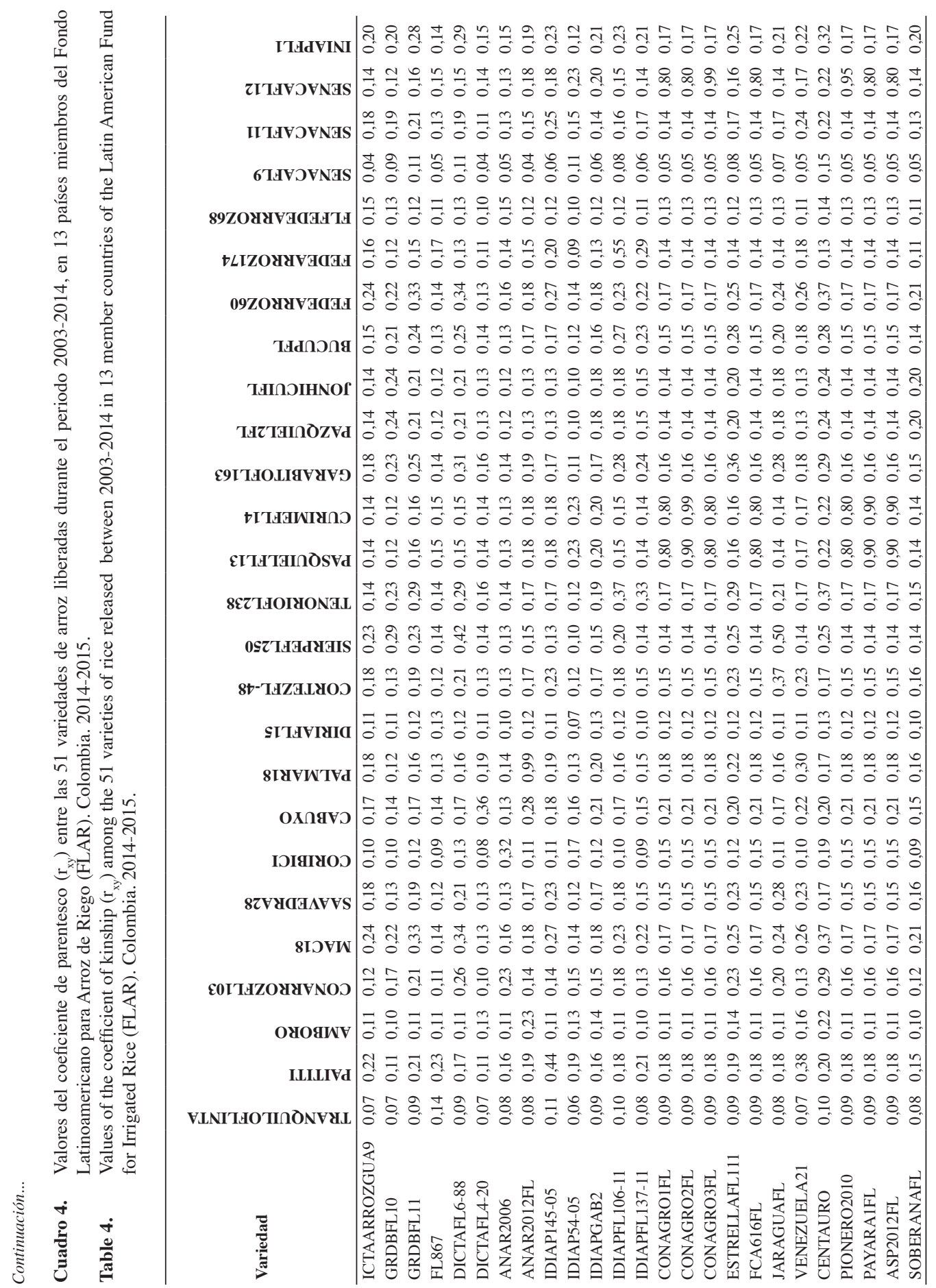




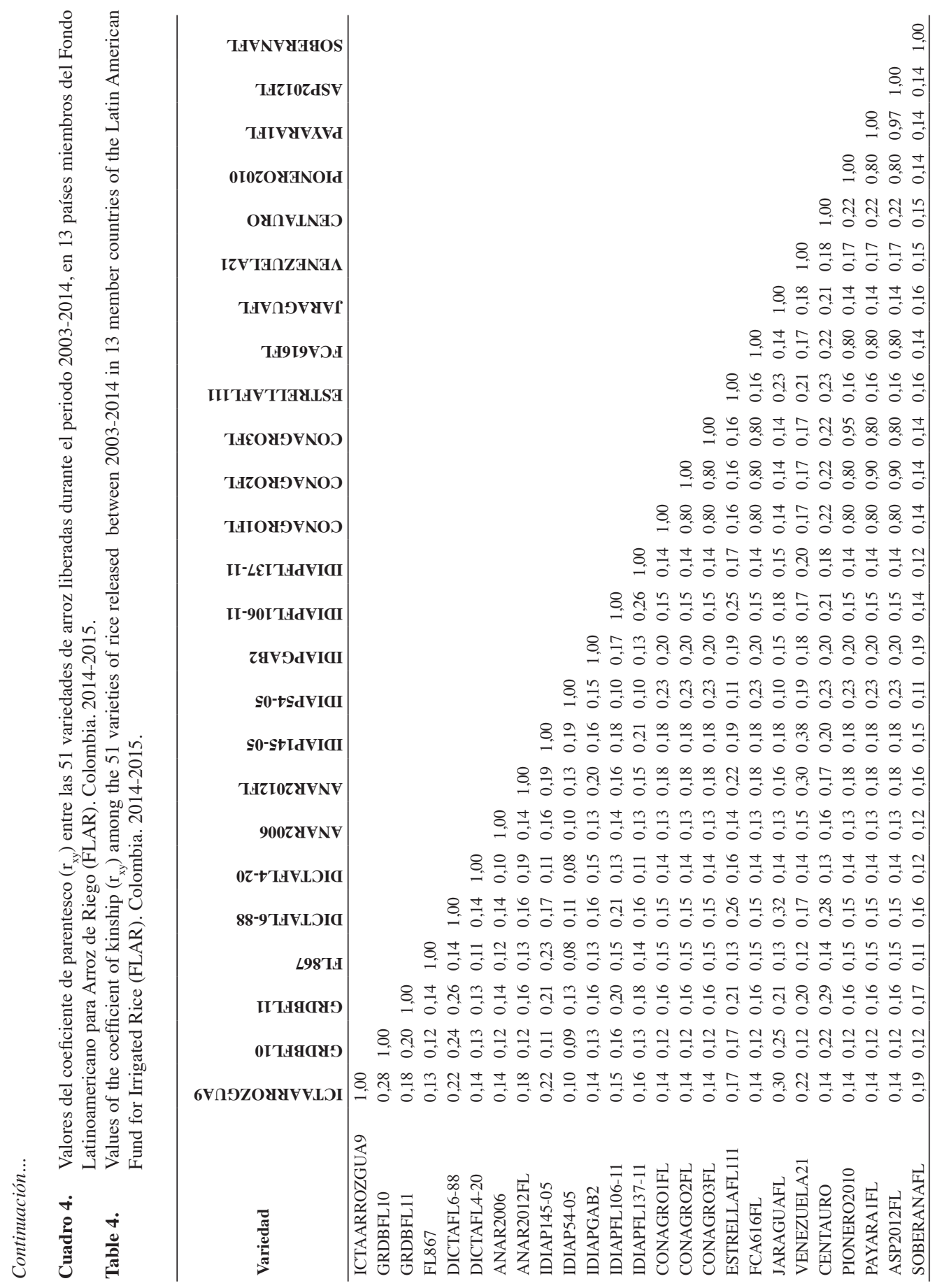




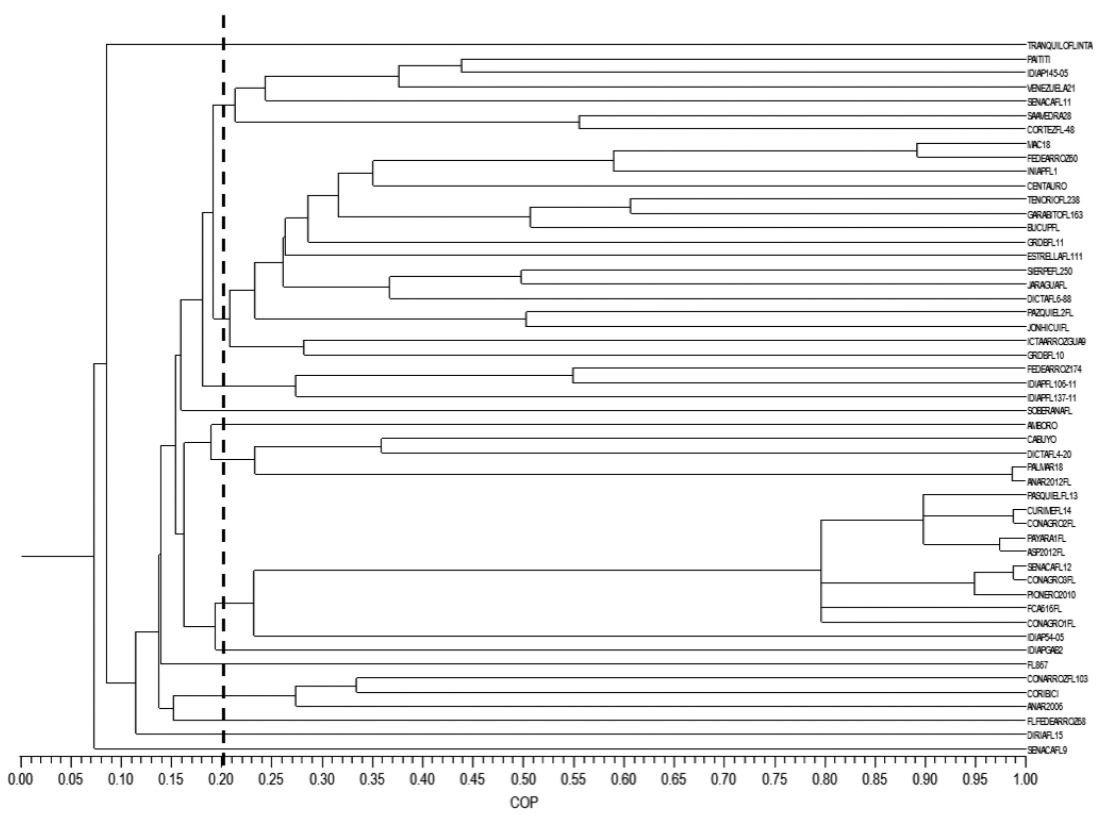

Figura 1. Dendograma basado en similaridad $\left(r_{x y}\right)$ entre 51 variedades de arroz liberadas durante el periodo 2003-2014, en 13 países miembros del Fondo Latinoamericano para Arroz de Riego (FLAR). Método UPGM. Colombia. 2014-2015.

Figure 1. Dendrogram based on similarity $\left(r_{x y}\right)$ among the 51 rice varieties released between 2003-2014 in 13 countries of the Latin American Fund for Irrigated Rice (FLAR). UPGM method. Colombia. 2014-2015.

Cuadro 5. Agrupación de las 51 variedades liberadas durante el periodo 2003-2014, en 13 países miembros del Fondo Latinoamericano de Arroz de Riego (FLAR), de acuerdo con su grado de parentesco. Colombia. 2014-2015.

Table 5. Grouping of the 51 varieties released between 2003-2014 in 13 countries of the Latin American Fund for Rice Irrigation (FLAR), according to their degree of kinship. Colombia. 2014-2015.

\begin{tabular}{ll}
\hline Grupo & Variedades \\
\hline I & Curime FL 14, CONAGRO 2 FL, CONAGRO 3 FL, SENACA FL 12, Payara 1 FL, ASP 2012 FL, Pionero 2010 FL, \\
& Pasquiel FL 13, CONAGRO 1 FL, FCA 616 FL e IDIAP 54-05. \\
\hline II & Palmar 18, ANAR 2012 FL, Cabuyo y DICTA Comayagua FL 4-20. \\
\hline III & $\begin{array}{l}\text { MAC 18, FEDEARROZ 60, Tenorio FL 238, Garabito FL 163, INIAP FL 01, Pasquiel 2 FL, Jonhiquí FL, Bu Cup FL, } \\
\text { Sierpe FL 250, Jaragua FL, DICTA Playitas FL 6-88, Centauro, GRDB FL 11, ICTA ARROZGUA 09, GRDB FL 10, } \\
\end{array}$ \\
\hline Estrella FL 111. \\
\hline IV & SAAVEDRA 28, Cortez FL 48, Paititi, IDIAP 145-05, Venezuela 21, SENACA FL 11. \\
\hline VI & FEDEARROZ 174, IDIAP FL 106-11, IDIAP FL 137-11. \\
\hline VII & IDIAPARROZ FL 103, Coribicí, ANAR 2006. \\
\hline VIII & Amboró. \\
\hline IX & Soberana FL. \\
\hline X & FL FEDEARROZ 68. \\
\hline XI & FL 867. \\
\hline XII & Diría FL 15. \\
\hline XIII & Tranquilo FL INTA. \\
\hline XIV & SENACA FL 09. \\
\hline
\end{tabular}


decir, se ha incrementado la variabilidad entre las nuevas variedades liberadas. Estos resultados fueron también similares para las variedades de Ecuador. Las variedades de arroz estudiadas constituyen un grupo heterogéneo de materiales de acuerdo con su parentesco, existen materiales bastante relacionados y otros muy poco relacionados.

Se observó un gran esfuerzo de los mejoradores de involucrar un mayor número de progenitores en las nuevas variedades, en busca de ampliar la base genética estrecha reportada por varios autores en el pasado. Esto es importante para el mejoramiento genético, ya que permitirá lograr mayor ganancia genética y posiblemente reducirá la vulnerabilidad a factores adversos.

La diversidad genética entre un grupo de cultivares puede ser estudiada utilizando diversas metodologías. Sin embargo, dado que el análisis del pedigrí, no se requiere realizar observaciones de tipo morfológico, molecular o de isoenzimas entre los genotipos a estudiar, además no está influenciada por el ambiente; es una metodología útil y económica que permite ofrecer una información preliminar acerca de las interrelaciones entre grupos de genotipos. Sin embargo, dadas las restricciones debidas a fallas en los supuestos en los que se basa este análisis, principalmente en que cada progenitor aporta el $50 \%$ de los genes, estos resultados constituyen una aproximación al análisis de la diversidad genética existente en variedades de arroz liberadas en los trece países de la región.

Estudios posteriores basados principalmente en análisis genéticos que utilicen marcadores moleculares permitirán obtener una visión más detallada de la estructura genética de las variedades de arroz de toda la región.

El tema de la vulnerabilidad genética en los cultivos y la estrecha base genética que impide mayores ganancias por selección, debe ser analizado cuidadosamente. El foco principal en el mejoramiento de una determinada especie, debe ser incorporar diversidad genética para resistencia a patógenos e insectos, y combinarla con homogeneidad para aquellos caracteres agronómicos y morfológicos que confieren altos rendimientos, adaptabilidad y estabilidad (Rajaran et al., 2002). Esto indica que aunque la uniformidad genética no necesariamente conduce a epidemias inmediatas, es deseable tener un conjunto génico más diverso entre los cultivares en uso. De esta forma, genes de resistencia originados de progenitores diversos introgresados en los cultivares elites, podrían ofrecer mayor protección contra apariciones inesperadas de plagas. Sin embargo, deben mantenerse varias características como arquitectura de la planta, resistencia a vuelco, tipo de grano y varios caracteres relacionados con calidad de grano.

\section{LITERATURA CITADA}

Acevedo, M., E. Torres, O. Moreno, W. Castrillo, O. Torres, R. Alvarez, E. Reyes, G. Torrealba, G. Salazar, y M. Navas. 2007. Base genética de los cultivares de arroz de riego liberados en Venezuela. Agron. Trop. 57:197-204.

Berrío-Orozco, L.E. 1999. Determinación de la variabilidad genética del arroz (Oryza sativa L.) desarrollado con germoplasma de africano. Tesis MSc., Universidad Nacional de Colombia, Palmira, COL.

Berrío Orozco, L.E., y E.P. Guimarães. 1995. Variabilidad Genética de las variedades de arroz liberadas en 1993 en América Latina y El Caribe. En: INGER, editor, Informe INGER América Latina 1994. CIAT, Palmira, COL. p. 56-65.

CIAT (Centro Internacional de Agricultura Tropical). 1987. Registro de cruzamientos realizados por los programas de arroz de ICA y del CIAT (1957-1986). CIAT, Cali, COL.

Cuevas-Pérez, F., E.P. Guimarães, L.E. Berrío, and D.I. González. 1992. Genetic base of irrigated-rice in Latin America and the Caribbean, 1971-1989. Crop Sci. 32:1054-1059.

Falconer, D.S., and T.F.C. Mackay. 1996. Introduction to quantitative genetics. 4 ed. Longman, Essex, GBR.

González-Tous, D.I., L.E. Berrio, N. Manosalva, y F. Cuevas. 1991. Origen de las variedades de arroz en Colombia 1971-1989. Arroz (Colombia) 42(372): 8-16.

Gopal, J., and K. Oyama. 2005. Genetic base of Indian potato selections as revealed by pedigree analysis. Euphytica 142:23-31.

IRIS (Internacional Rice information System). 2011. Breeding Management System (BMS), Generation Challengue Program, CGIAR. https://www.integratedbreeding.net/ breeding-management-system (accessed 2013-2014).

Manosalva de Nivia, L.E. Berrio, D.I. González, y F. Cuevas. 1991. Variabilidad genética del arroz en América Central. Trabajo presentado durante la 
XXXVII Reunión Anual del Programa Cooperativo Centroamericano para el Mejoramiento de Cultivos y Animales (PCCMCA). 18-22 marzo 1991. PAN.

Rajaran, S., N.E. Bourlaug, and M. Van Guinkel. 2002. CIMMYT international wheat breeding. In: B.C. Curtis et al., editors, Bread wheat: improvement and Production. FAO. Roma, ITA. p. 103-118.

Rangel P., E. Guimarães, e P. Neves. 1996. Base genética das cultivares de arroz (Oryza sativa L.) irrigado do Brasil. Pesq. Agropec. Bras. 31:349-357.

Rohlf, F.J. 1997. NTSYS-pc. Numerical taxonomy and multivariate analysis system. Version 2.2e. Exeter Software, NY, USA.
Tanksley, S.D., and J.C. Nelson. 1996. Advanced backcross QTL analysis: a method for the simultaneous discovery and transfer of valuable QTLs from unadapted germplasm into elite breeding lines. Theor. Appl. Genet. 92:191-203.

Vello, N.A., D.M. Hiromoto, and A.J.B.V. Azevedo-Filho. 1988. Coefficient of parentage and breeding of Brazilian soybean germplasm. Rev. Bras. Genét. 11:679-697.

Wang, Z.W., G. Second, and S.D. Tanksley. 1992. Polymorphism and phylogenetic relationships among species in the genus Oryza as determined by analysis of nuclear RFLPs. Theor. Appl. Genet. 83:565-581. 